\title{
Diagnostic value of circular RNAs in female reproductive system diseases: A PRISMA-compliant meta-analysis
}

\author{
JIN DING, YUANYUAN LYU, NAN GUO, QINGWEI WANG, LINA LI and GUANTAI NI \\ Department of Obstetrics and Gynecology, The First Affiliated Hospital of Wannan Medical College, \\ Wuhu, Anhui 241001, P.R. China
}

Received September 18, 2019; Accepted December 31, 2019

DOI: $10.3892 /$ br.2020.1278

\begin{abstract}
Circular RNAs (circRNAs) are novel non-coding RNAs that have been reported to be involved in the progression of numerous diseases. However, the clinical diagnostic value of circRNAs in female reproductive system diseases remains unknown. The present study is a systemic review and meta-analysis of the available literature on circRNAs as novel biomarkers for female reproductive system diseases. Relevant studies were systematically searched using the PubMed, Embase, Web of Science and Cochrane Library databases. The data obtained from the included studies were analyzed by RevMan5.3 and STATA 14.2. A total of six studies involving 613 individuals across three types of disease examined the diagnostic capabilities of circRNAs. Within these publications, the pooled sensitivity of circRNAs was 0.70 (95\% CI, 0.64-0.76), and the pooled specificity was 0.70 (95\% CI, 0.64-0.75). The pooled positive likelihood ratio and negative likelihood ratio were 2.33 and 0.42 , respectively. The diagnostic score was 1.70 and the pooled diagnostic odds ratio was 5.48. The area under the summary receiver operator characteristic curve was 0.76 (95\% CI, 0.72-0.79), indicating that circRNAs exhibited a moderate diagnostic value for female reproductive system diseases and may function as potential diagnostic biomarkers. However, further studies are required to verify the clinical applications of circRNAs.
\end{abstract}

\section{Introduction}

A number of female reproductive system diseases such as repeated implantation failure, preeclampsia, endometriosis, reproductive system malignancy and breast cancer lack tools for early diagnosis. Although clinical biomarkers such as squamous cell carcinoma antigen and CA125/199 can be used in the diagnosis

Correspondence to: Dr Guantai Ni, Department of Obstetrics and Gynecology, The First Affiliated Hospital of Wannan Medical College, 2 West Zheshan Road, Wuhu, Anhui 241001, P.R. China

E-mail: ngtiger@aliyun.com

Key words: circular RNA; diagnostic biomarker; meta-analysis; female reproductive system diseases of female reproductive tract tumors, they are of low sensitivity and specificity $(1,2)$. Therefore, new effective biomarkers are urgently needed for the diagnosis of these conditions.

Non-coding RNAs have been extensively used in clinical experiments and are of great potential as biomarkers for detection of disease (3). Circular RNAs (circRNAs) are a class of endogenous non-coding RNA and consist of a covalently closed continuous loop with neither 5'-3' polarity nor a polyadenylated tail $(4,5)$. Unlike linear RNAs, circRNAs are protected against the effects of RNA enzymes due to their lack of free ends; these molecules are thus more stable compared with linear RNAs (5). With the development of RNA-seq analysis and bioinformatics technologies, recent studies have reported the use of circRNAs in the early detection and prognosis of certain types of cancer, such as gastric cancer (6), hepatocellular carcinoma (7), lung cancer (8), cervical squamous cell carcinoma (CSCC) and breast cancer (BRCA) (9), as well as a number of female reproductive system diseases including repeated implantation failure (10), preeclampsia (11) and ovarian endometriosis (12). Considering the association between hormone-responsive BRCA and the female reproductive system (13), this condition was included as a female reproductive system disease in the present meta-analysis.

The aim of this meta-analysis was to summarize all circRNAs that have been investigated as diagnostic markers for female reproductive system diseases and to review their efficiency as novel diagnostic biomarkers in such diseases. Available data from published literature were evaluated to determine if circRNAs may be used as sensitive and specific molecular biomarkers.

\section{Materials amd methods}

Search strategy. This systematic meta-analysis was performed in strict accordance with the guidelines for diagnostic meta-analysis. Eligible studies published on PubMed (https://www.ncbi.nlm.nih.gov/pubmed), EMBASE (https://embase.com/), Web of Science (https://www. isiknowledge.com/) and Cochrane Library (https://www. cochranelibrary.com/) before February 20, 2019 were selected for meta-analysis. Only studies published in English were included. No restrictions were applied for the year of publication or publication status. Databases were search using the following keywords: 'Circular RNA' OR 'circRNA' AND 


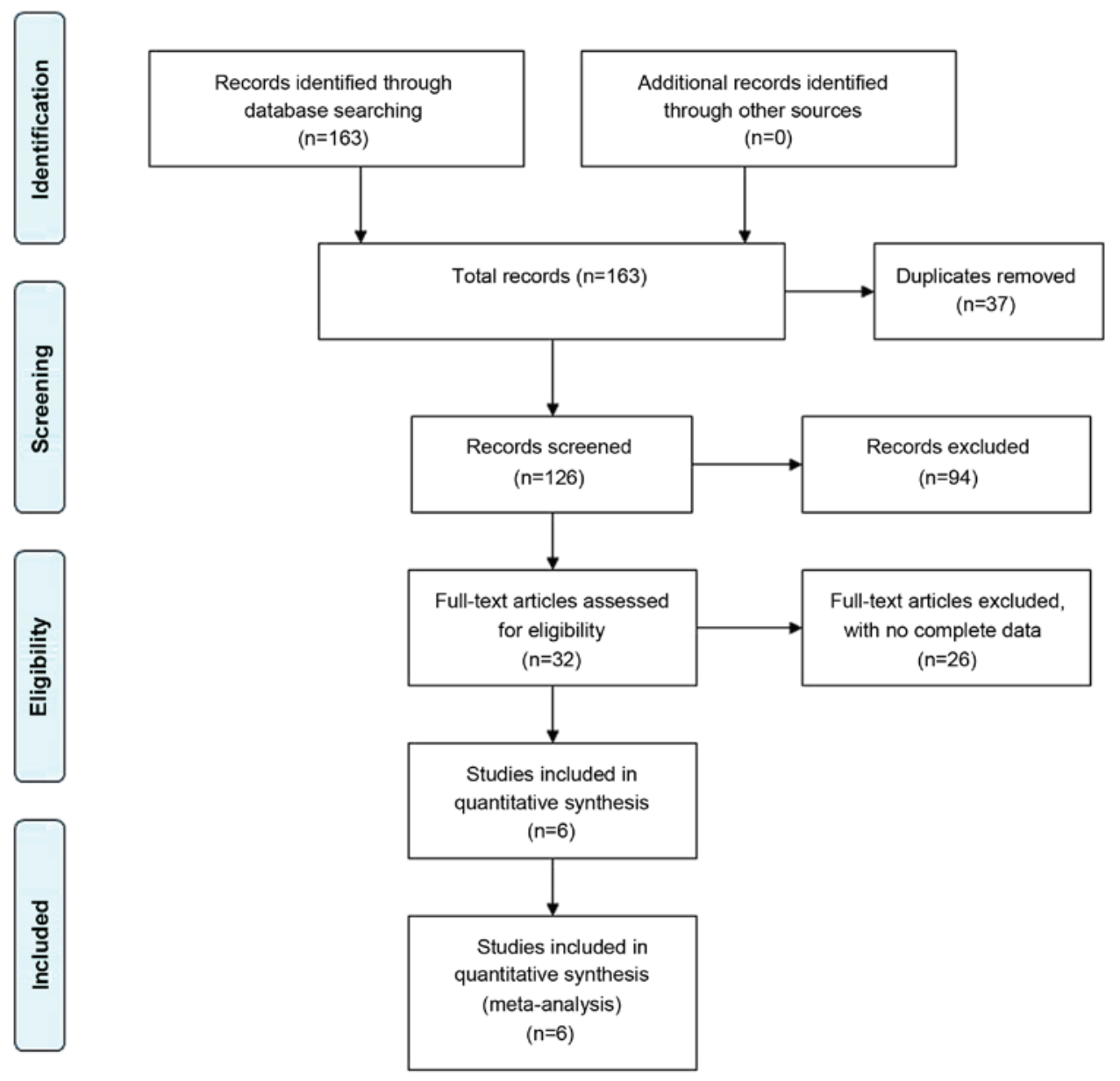

Figure 1. Flowchart of the search strategy.

'endometrial' OR ‘endometrium' OR 'ovarian' OR 'ovary' OR 'cervical' OR ‘uterine' OR 'uterus' OR 'uterine cervix' OR 'breast' OR 'vagina*' OR 'pregnancy' OR 'pre-eclampsia' OR 'PCOS' OR ‘placenta previa' OR 'gynaecology' OR 'obstetrics' OR 'genitalia', and 'diagnosis' OR 'diagnostic' OR 'sensitivity' OR 'specificity' OR 'receiver operating characteristic curve' OR 'ROC' OR 'AUC'.

Selection of publications. Two researchers independently reviewed all search results based on the titles and abstracts; the relevant studies were included in the full text review. Data extraction was performed by other researchers. Any disagreement regarding the inclusion or exclusion of studies were resolved by discussion involving a third investigator. All studies included in the meta-analysis met the following criteria: i) Studies that reported on the diagnostic value of circRNAs for any female reproductive system diseases type; ii) studies which contained sample, sensitivity, specificity and AUC data; and iii) studies that enrolled $>30$ cases and matched controls. Studies were excluded as follows: i) Duplicate studies; ii) reviews, letters, conference abstracts, case reports and articles with insufficient data; iii) articles studying circRNA in cell lines; and iv) articles published in languages other than English.

Data extraction and quality assessment. The following parameters were obtained from all studies: First author name, publication year, study area, patient ethnicity, disease type, specimen, sample size, as well as data on circRNA sensitivity and specificity. The Quality Assessment of Diagnostic Accuracy Studies-2 (QUADAS-2) tool (14) was used to perform quality assessment of each included study.

Statistical analysis. All statistical analyses were conducted using the analytical software RevMan5.3 (The Cochrane Collaboration) and STATA14.2 (StataCorp LLC). All data such as the number of true positives (TP), false positives (FP), true negatives $(\mathrm{TN})$ and false negatives $(\mathrm{FN})$ were extracted from each study to calculate the pooled sensitivity and specificity, positive likelihood ratio (PLR), negative likelihood ratio (NLR), diagnostic odds ratio (DOR) and their $95 \%$ confidence intervals (CI), summary receiver operator characteristic (sROC) curve and area under the curve (AUC). The data obtained was used to determine the overall performance of circRNAs in identifying female reproductive system diseases. $\mathrm{P}<0.05$ was considered to indicate a statistically significant difference. In addition, heterogeneity across studies was determined using Cochran's Q and $\mathrm{I}^{2}$ statistics, where $\mathrm{I}^{2}>50 \%$ indicated the existence of significant heterogeneity. Meta-regression analysis was utilized to detect the possible sources of heterogeneity.

\section{Results}

Literature search. A total of 163 potentially eligible articles were reviewed in this meta-analysis. The literature search 


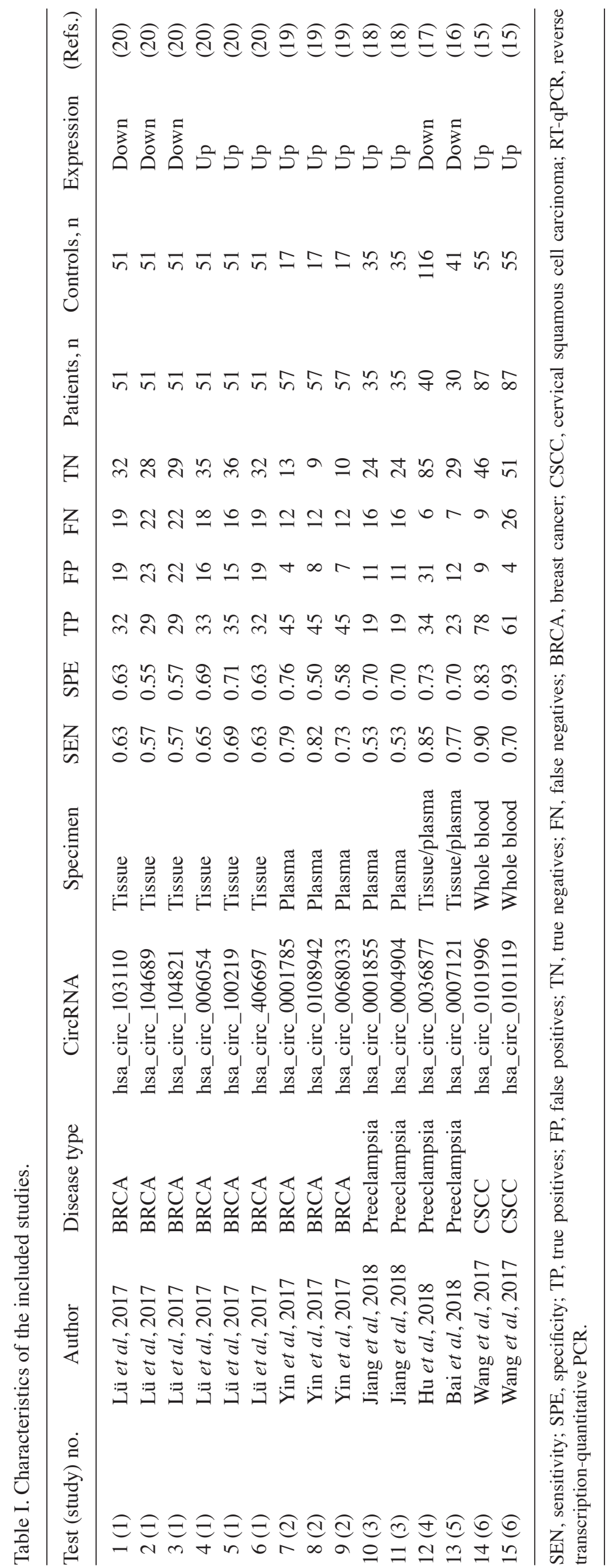




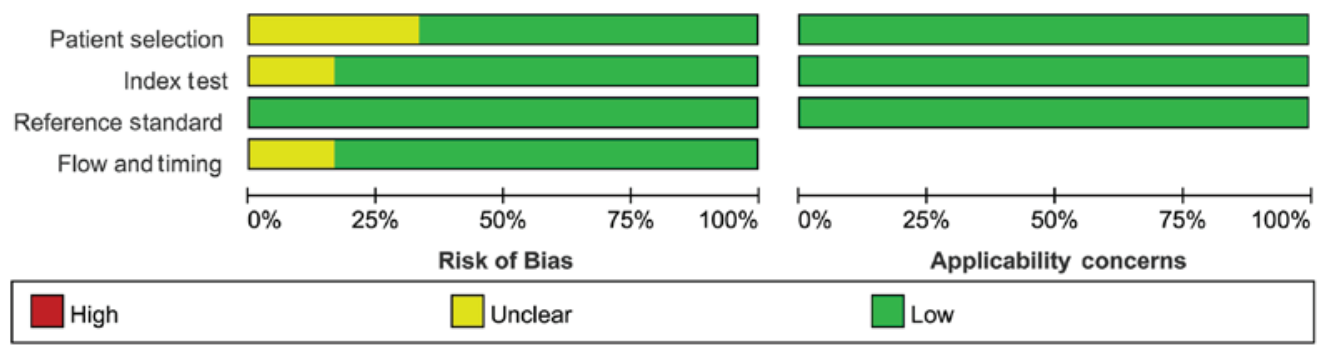

Figure 2. Methodological quality graph.

A

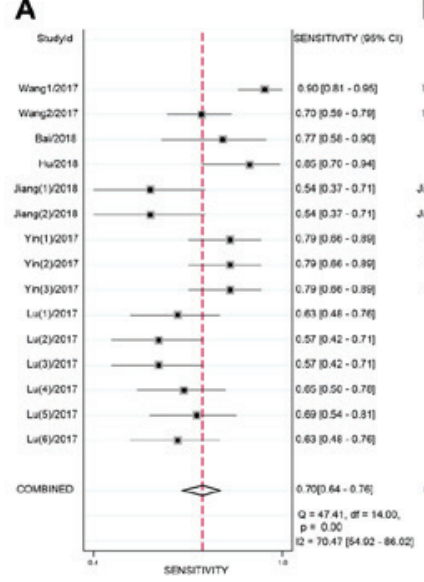

B

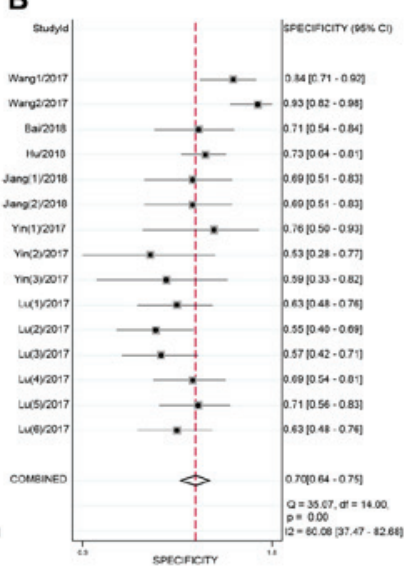

C

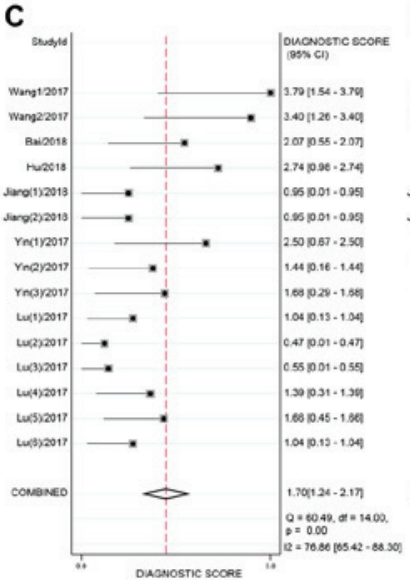

D

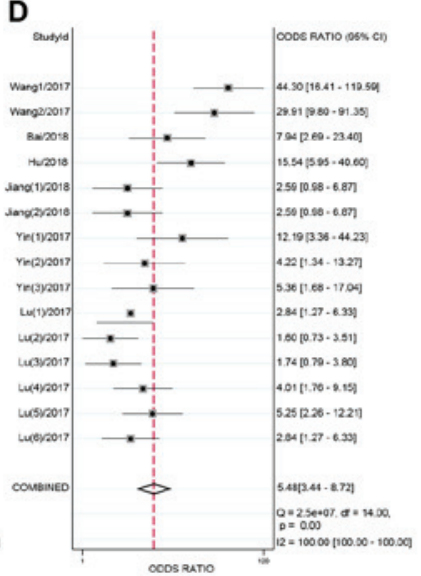

$\mathbf{E}$
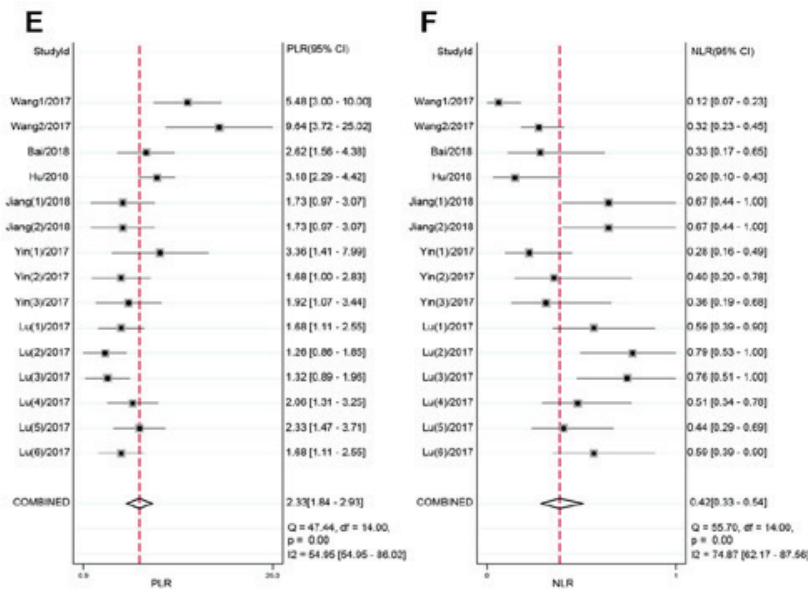

G

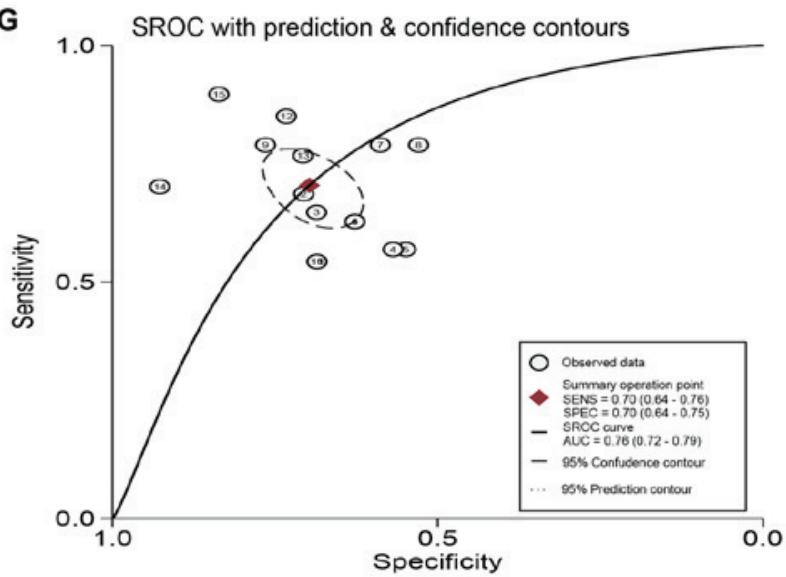

Figure 3. Forest plots of (A) sensitivity; (B) specificity; (C) PLR; (D) NLR; (E) diagnostic score; (F) DOR; and (G) AUC. PLR, positive likelihood ratio; NLR, negative likelihood ratio; DOR, diagnostic odds ratio; AUC, area under the curve; SROC, summary receiver operator characteristic; SENS, sensitivity; SPEC, specificity.

strategy is depicted as a flow chart in Fig. 1. Among the 163 studies, 37 were duplicates and 22 were reviews, letters or conference abstracts. Following screening the titles and abstracts of the remaining publications, 70 were identified not to be relevant to the present study. A total of 34 articles were eligible for full-text review, and 28 articles were excluded due to incomplete full-texts or incomplete data. Finally, six eligible studies (each circRNA as a test, a total of 15 tests) were included in the meta-analysis (15-20).

Study characteristics. The characteristics of the six included studies with 15 tests are summarized in Table I: A total of 613 individuals representing three types of disease were enrolled in the selected studies. These studies were of high quality based on the QUADAS-2 analysis (Fig. 2).
Meta-analysis. Overall, the detection performance of circRNAs was as follows: The pooled sensitivity was 0.70 (95\% CI, 0.64-0.76; $\mathrm{Q}=47.41 ; \mathrm{P}<0.001 ; \mathrm{I}^{2}=70.47 \%$ ) and the pooled specificity was $0.70(95 \% \mathrm{CI}, 0.64-0.75 ; \mathrm{Q}=35.07$; $\left.\mathrm{P}<0.001 ; \mathrm{I}^{2}=60.08 \%\right)$. The PLR and NLR were $2.33(\mathrm{Q}=47.44$; $\left.\mathrm{P}=0.001 ; \mathrm{I}^{2}=54.95 \%\right)$ and $0.42(95 \% \mathrm{CI}, 0.33-0.54 ; \mathrm{Q}=55.70$; $\left.\mathrm{P}<0.001 ; \mathrm{I}^{2}=74.87 \%\right)$, respectively, and the diagnostic score was $1.70\left(\mathrm{Q}=60.49 ; \mathrm{P}<0.001 ; \mathrm{I}^{2}=76.86 \%\right)$. The pooled $\mathrm{DOR}$ was $5.48\left(\mathrm{Q}=2.5 \times 10^{7} ; \mathrm{P}<0.001 ; \mathrm{I}^{2}=100.00 \%\right)$. Additionally, the AUC of the sROC curve was 0.76 (95\% CI, 0.72-0.79). The relevant forest plots and sROC are presented in Fig. 3.

Meta-regression analyses. A meta-regression based on disease type (benign or malignant) and sample size $(>100$ or $\leq 100)$ was used to identify the possible sources of heterogeneity. 
Table II. Meta-regression analysis.

\begin{tabular}{|c|c|c|c|c|c|c|}
\hline Parameter & Category & No. of studies & Sensitivity (95\% CI) & $\mathrm{P} 1$ & Specificity $(95 \%$ CI $)$ & $\mathrm{P} 2$ \\
\hline \multirow[t]{2}{*}{ Disease type } & Malignant & 11 & $0.71(0.64-0.78)$ & 0.17 & $0.70(0.63-0.76)$ & $0.04^{\mathrm{a}}$ \\
\hline & Benign & 4 & $0.68(0.56-0.81)$ & & $0.70(0.60-0.80)$ & \\
\hline \multirow[t]{2}{*}{ Sample size } & $\geq 100$ samples & 9 & $0.70(0.62-0.77)$ & $0.02^{\mathrm{a}}$ & $0.70(0.64-0.77)$ & 0.08 \\
\hline & $<100$ samples & 6 & $0.72(0.63-0.81)$ & & $0.68(0.58-0.78)$ & \\
\hline
\end{tabular}

${ }^{\text {aP }}<0.05$. P1, sensitivity P-value; P2, specificity P-value.

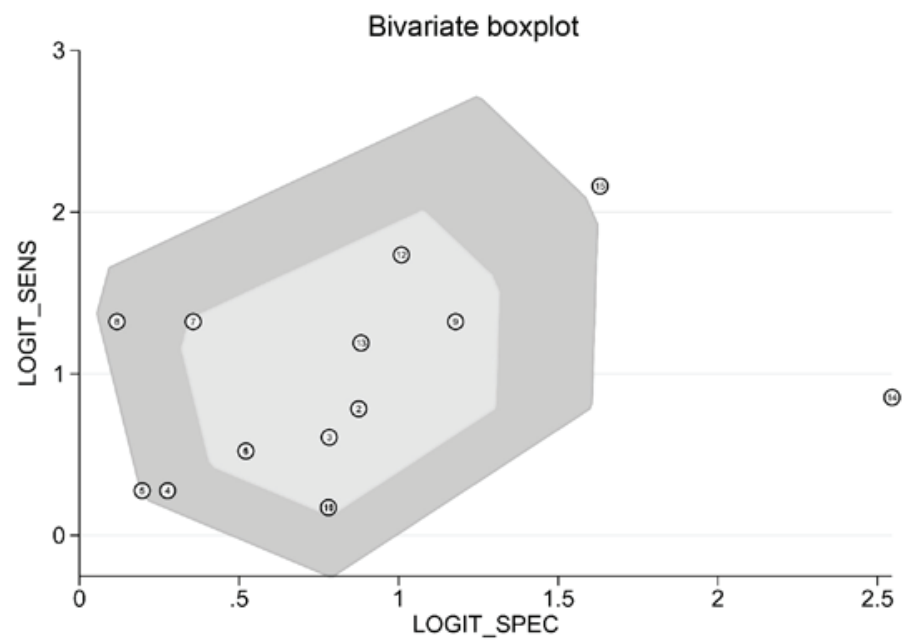

Figure 4. Bivariate boxplot. Bivariate boxplot was based on the SENS and SPEC of the tests, which is a useful tool for the detection of heterogeneity. Tests not located in the boxplot included test 14 and 15, which belonged study no. 6 (cervical squamous cell carcinoma), indicating that they may have affected the heterogeneity. SENS, sensitivity; SPEC, specificity.

The results of the meta-regression analysis of probable factors suggested that different sample sizes may increase heterogeneity in pooled sensitivity $(\mathrm{P}=0.02)$, and disease types may result in heterogeneity in pooled specificity $(\mathrm{P}=0.04)$ (Table II). The bivariate boxplot demonstrates the heterogeneity of each study (Fig. 4). Two tests not included in the boxplot belonged study no. 6 (CSCC group). After excluding this study, Cochran's $\mathrm{Q}$ and $\mathrm{I}^{2}$ were decreased in the resultant forest plot, which was indicative of improvement in homogeneity. However, the sensitivity decreased from 0.70 to 0.68 , specificity decreased from 0.70 to 0.66 , PLR decreased from 2.33 to 2.20 , NLR increased from 0.42 to 0.48 and sROC decreased from 0.76 to 071 (Fig. 5). This reduction suggested that study 6 (CSCC group) had high sensitivity and specificity compared with the other studies.

\section{Discussion}

CircRNA is a novel category of non-coding RNAs with a closed circular structure. These specialized structures make circular RNAs more stable than linear RNAs due to their resistance to RNA enzymes such as exonuclease and ribonuclease (5). A previous study has demonstrated that the half-life of mRNAs is only about $10 \mathrm{~h}$, whereas whole circRNAs have a half-life $>48 \mathrm{~h}$ (20). CircRNAs are highly abundant in various human tissues and cell samples and exhibit highly tissue-specific expression, especially in hepatocellular, cervical and ovarian carcinoma $(21,22)$. For these reasons, circRNA are excellent candidate biomarkers for diagnosing human diseases.

In the present meta-analysis, relevant articles were screened across four databases, and six relevant studies were finally included to evaluate the diagnostic value of circRNAs in diseases of the female reproductive system. To the best of our knowledge, this is the first meta-analysis performed on this topic. The pooled sensitivity and specificity were 0.70 and 0.70 , respectively, indicating that circRNAs may be valid diagnostic markers in female reproductive system diseases. The pooled DOR was 5.48, and the AUC of the sROC curve was 0.76 . These results demonstrated that circRNAs exhibited a moderate diagnostic performance. Similarly, circRNAs have been reported to possess prognostic value for female reproductive system tumors, such as endometrial and epithelial ovarian cancer $(23,24)$, but no specific diagnostic data was available. Overall, circRNAs may be appropriate for use as diagnostic biomarkers in female reproductive system diseases.

However, it should be highlighted that heterogeneity was present in the current pooled estimates, as the included studies involved experiments which used whole blood, plasma or tissues. A meta-regression based on disease type and sample size was performed; the results demonstrated that the heterogeneity may arise from the sample size and disease type. The bivariate boxplot demonstrated that study no. 6 (regarding CSCC) was the source of heterogeneity. Exclusion of this study resulted in decreased $\mathrm{Q}$ and $\mathrm{I}^{2}$ values, both of which are 
A

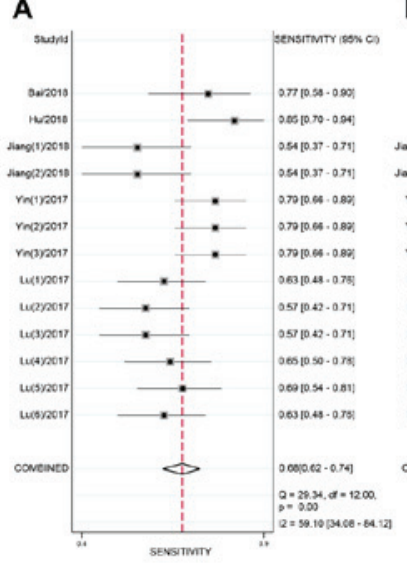

B

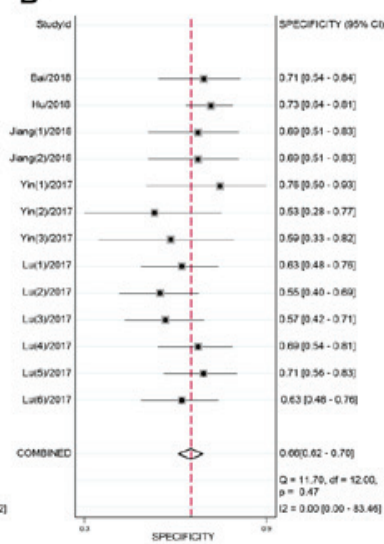

C

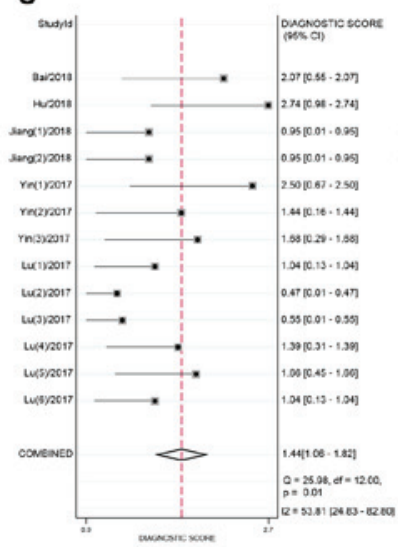

D

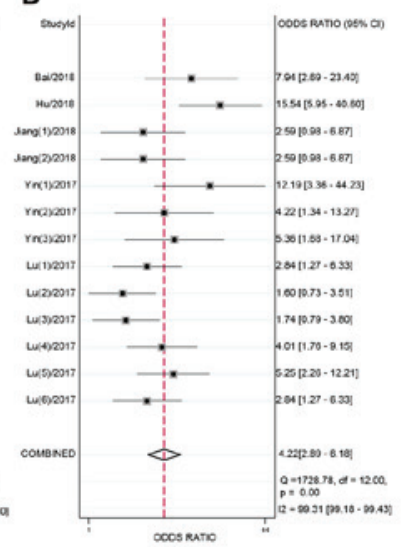

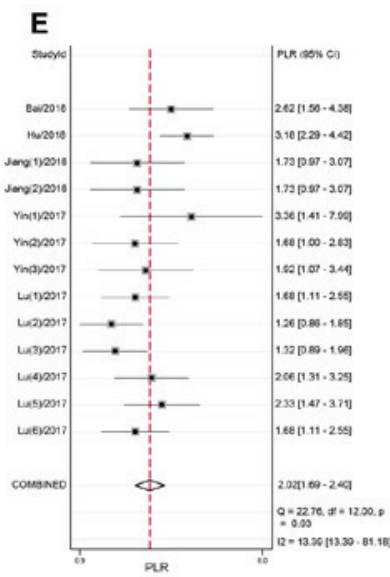

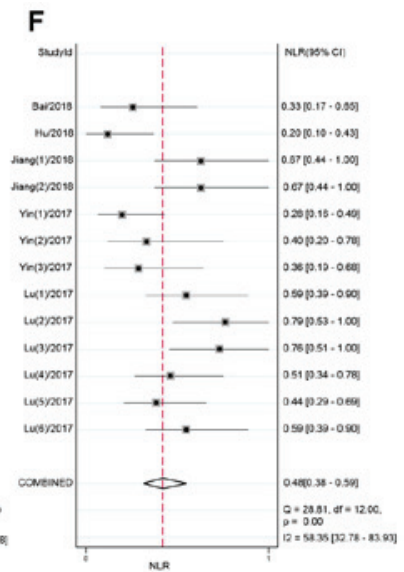

G

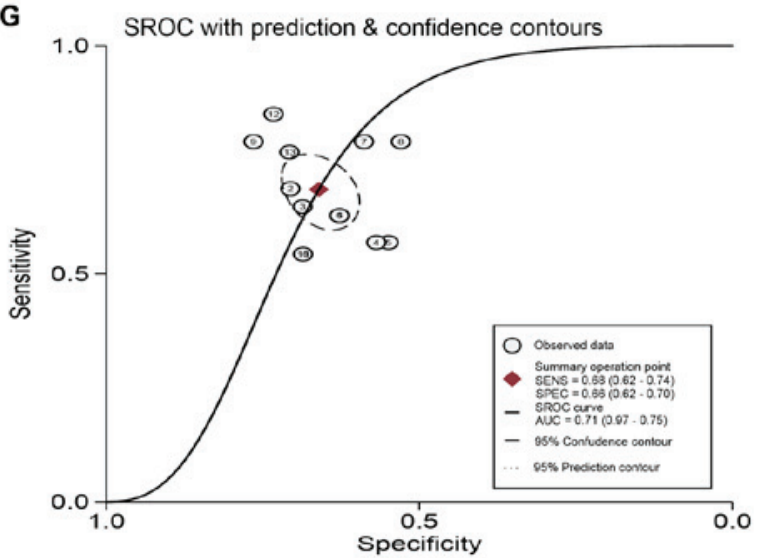

Figure 5. After excluding the CSCC group, forest plots of (A) sensitivity; (B) specificity; (C) PLR; (D) NLR; (E) diagnostic score; (F) DOR; and (G) AUC. CSCC, cervical squamous cell carcinoma; PLR, positive likelihood ratio; NLR, negative likelihood ratio; DOR, diagnostic odds ratio; AUC, area under the curve; SROC, summary receiver operator characteristic; SENS, sensitivity; SPEC, specificity.

indicators of disease heterogeneity. Study no. 6 was the only study on cervical cancer which utilized whole blood, which may have been the source of heterogeneity.

The limitations of this meta-analysis should be taken into consideration. Firstly, all included studies were authored by Chinese investigators and were on ethnic Chinese patient samples. Therefore, the overall diagnostic accuracy of circRNAs may be not be applicable to the general population; future research regarding circRNAs as biomarkers should be expanded to multiple countries and ethnicities. Next, the enrolled studies only included data on those with preeclampsia, CSCC and BRCA. There are several other female reproductive system diseases that lack early diagnostic tools including repeated implantation failure and reproductive system malignancy such as ovarian cancer, the 5-year survival rate of which is only $30 \%$ as a vast majority of patients present with widespread metastasis $(25,26)$. The lack of an effective molecular biomarker for diagnosing early stage ovarian cancer is a key contributor to its overall poor prognosis (27). Lastly, tissue-extracted circRNAs may not be the ideal biomarker considering that circRNAs are also stable and abundant in exosomes $(28,29)$. The detection of circRNAs from exosomes in plasma or serum may be a better alternative in diagnosing disease. The results of the present meta-analysis suggested that circRNAs may serve as a useful, noninvasive molecular biomarker for clinical practice in the future. More extensive studies are urgently needed to evaluate the diagnostic performance of plasma or serum circRNAs in the context of female reproductive system diseases.

In conclusion, circRNAs possess the potential to function as diagnostic biomarkers for female reproductive system diseases. Additional large-scale studies are required to verify the results of this preliminary study.

\section{Acknowledgements}

Not applicable.

\section{Funding}

The present study was supported by The Open Project of The Key Laboratory of Non-coding RNA Transformation Research of Anhui Higher Education Institution (Wannan Medical College; grant no. RNA201901).

\section{Availability of data and materials}

All data generated or analyzed during this study are included in this published article.

\section{Authors' contributions}

JD and GN designed the study and prepared the manuscript with comments from all authors. JD and YL performed data 
extraction and quality assessment. JD wrote and revised the manuscript. NG, QW and LL drafted the manuscript and critically revised it for important intellectual content. All authors read and approved the final manuscript.

\section{Ethics approval and consent to participate}

Not applicable.

\section{Patient consent for publication}

Not applicable.

\section{Competing interests}

The authors declare that they have no competing interests.

\section{References}

1. Zhao S, Mei Y, Wang Y, Zhu J, Zheng G and Ma R: Levels of CEA, CA153, CA199, CA724 and AFP in nipple discharge of breast cancer patients. Int J Clin Exp Med 8: 20837-20844, 2015

2. Wang HF, Li LF, Guo SH, Zeng QY, Ning F, Liu WL and Zhang G: Evaluation of antibody level against Fusobacterium nucleatum in the serological diagnosis of colorectal cancer. Sci Rep 6: 33440, 2016.

3. Beermann J, Piccoli MT, Viereck J and Thum T: Non-coding RNAs in development and disease: Background, mechanisms, and therapeutic approaches. Physiol Rev 96: 1297-1325, 2016.

4. Salzman J: Circular RNA expression: Its potential regulation and function. Trends Genet 32: 309-316, 2016.

5. Hsiao KY, Sun HS and Tsai SJ: Circular RNA-New member of noncoding RNA with novel functions. Exp Biol Med (Maywood) 242: 1136-1141, 2017.

6. Shen F, Liu P, Xu Z, Li N, Yi Z, Tie X, Zhang Y and Gao L: CircRNA_001569 promotes cell proliferation through absorbing miR-145 in gastric cancer. J Biochem 165: 27-36, 2019.

7. Han D, Li J, Wang H, Su X, Hou J, Gu Y, Qian C, Lin Y, Liu X, Huang M, et al: Circular RNA circMTO1 acts as the sponge of microRNA-9 to suppress hepatocellular carcinoma progression. Hepatology 66: 1151-1164, 2017.

8. Qu D, Yan B, Xin R and Ma T: A novel circular RNA hsa circ_0020123 exerts oncogenic properties through suppression of miR-144 in non-small cell lung cancer. Am J Cancer Res 8: $1387-1402,2018$

9. Zhang HD, Jiang LH, Hou JC, Zhou SY, Zhong SL, Zhu LP, Wang DD, Yang SJ, He YJ, Mao CF, et al: Circular RNA hsa_circ 0072995 promotes breast cancer cell migration and invasion through sponge for miR-30c-2-3p. Epigenomics 10 : $1229-1242,2018$

10. Liu L, Li L, Ma X, Yue F, Wang Y, Wang L, Jin P and Zhang X Altered circular RNA expression in patients with repeated implantation failure. Cell Physiol Biochem 44: 303-313, 2017.

11. Zhang YG, Yang HL, Long Y and Li WL: Circular RNA in blood corpuscles combined with plasma protein factor for early prediction of pre-eclampsia. BJOG 123: 2113-2118, 2016

12. Xu XX, Jia SZ, Dai Y, Zhang JJ, Li XY, Shi JH, Leng JH and Lang JH: Identification of circular RNAs as a novel biomarker for ovarian endometriosis. Chin Med J (Engl) 131: 559-566, 2018

13. Nagini S: Breast Cancer: Current molecular therapeutic targets and new players. Anticancer Agents Med Chem 17: 152-163, 2017.

14. Whiting PF, Rutjes AW, Westwood ME, Mallett S, Deeks JJ, Reitsma JB, Leeflang MM, Sterne JA and Bossuyt PM; QUADAS-2 Group: QUADAS-2: A revised tool for the quality assessment of diagnostic accuracy studies. Ann Intern Med 155: $529-536,2011$.
15. Wang YM, Huang LM, Li DR, Shao JH, Xiong SL, Wang CM and Lu SM: Hsa_circ_0101996 combined with hsa_circ_0101119 in peripheral whole blood can serve as the potential biomarkers for human cervical squamous cell carcinoma. Int J Clin Exp Pathol 10: 11924-11931, 2017.

16. Bai Y, Rao H, Chen W, Luo X, Tong C and Qi H: Profiles of circular RNAs in human placenta and their potential roles related to preeclampsia. Biol Reprod 98: 705-712, 2018.

17. Hu X, Ao J, Li X, Zhang H, Wu J and Cheng W: Competing endogenous RNA expression profiling in pre-eclampsia identifies hsa circ 0036877 as a potential novel blood biomarker for early pre-eclampsia. Clin Epigenetics 10: 48, 2018.

18. Jiang $\mathrm{M}$, Lash GE, Zhao $\mathrm{X}$, Long $\mathrm{Y}$, Guo $\mathrm{C}$ and Yang $\mathrm{H}$ CircRNA-0004904, CircRNA-0001855, and PAPP-A: Potential novel biomarkers for the prediction of preeclampsia. Cell Physiol Biochem 46: 2576-2586, 2018.

19. Yin WB, Yan MG, Fang X, Guo JJ, Xiong W and Zhang RP: Circulating circular RNA hsa_circ_0001785 acts as a diagnostic biomarker for breast cancer detection. Clin Chim Acta 487: 363-368, 2018.

20. Lü L, Sun J, Shi P, Kong W, Xu K, He B, Zhang S and Wang J: Identification of circular RNAs as a promising new class of diagnostic biomarkers for human breast cancer. Oncotarget 8: 44096-44107, 2017.

21. Jeck WR and Sharpless NE: Detecting and characterizing circular RNAs. Nat Biotechnol 32: 453-461, 2014.

22. Gao YL, Zhang MY, Xu B, Han LJ, Lan SF, Chen J, Dong YJ and Cao LL: Circular RNA expression profiles reveal that hsa circ_0018289 is up-regulated in cervical cancer and promotes the tumorigenesis. Oncotarget 8: 86625-86633, 2017.

23. Chen Q, Zhang J, He Y and Wang Y: hsa_circ_0061140 knockdown reverses FOXM1-mediated cell growth and metastasis in ovarian cancer through miR-370 sponge activity. Mol Ther Nucleic Acids 13: 55-63, 2018.

24. Chen BJ, Byrne FL, Takenaka K, Modesitt SC, Olzomer EM, Mills JD, Farrell R, Hoehn KL and Janitz M: Analysis of the circular RNA transcriptome in endometrial cancer. Oncotarget 9: 5786-5796, 2017

25. Ning L, Long B, Zhang W, Yu M, Wang S, Cao D, Yang J, Shen K, Huang Y and Lang J: Circular RNA profiling reveals circEXOC6B and circN4BP2L2 as novel prognostic biomarkers in epithelial ovarian cancer. Int J Oncol 53: 2637-2646, 2018.

26. Bartl T, Schwameis R, Stift A, Bachleitner-Hofmann T, Reinthaller A, Grimm C and Polterauer S: Predictive and prognostic implication of bowel resections during primary cytoreductive surgery in advanced epithelial ovarian cancer. Int J Gynecol Cancer 28: 1664-1671, 2018.

27. AhmedI,Karedath T, Andrews SS, Al-Azwani IK, Mohamoud YA, Querleu D, Rafii A and Malek JA: Altered expression pattern of circular RNAs in primary and metastatic sites of epithelial ovarian carcinoma. Oncotarget 7: 36366-36381, 2016.

28. Hang W, Feng Y, Sang Z, Yang Y, Zhu Y, Huang Q and Xi X: Downregulation of miR-145-5p in cancer cells and their derived exosomes may contribute to the development of ovarian cancer by targeting CT. Int J Mol Med 43: 256-266, 2019.

29. Hou J, Jiang W, Zhu L, Zhong S, Zhang H, Li J, Zhou S, Yang S, He Y, Wang D, et al: Circular RNAs and exosomes in cancer: A mysterious connection. Clin Transl Oncol 20: 1109-1116, 2018.

30. Li S, Li Y, Chen B, Zhao J, Yu S, Tang Y, Zheng Q, Li Y, Wang P, He X and Huang S: ExoRBase: A database of circRNA, lncRNA and mRNA in human blood exosomes. Nucleic Acids Res 46: D106-D112, 2018.

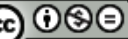

This work is licensed under a Creative Commons Attribution-NonCommercial-NoDerivatives 4.0 International (CC BY-NC-ND 4.0) License. 